\title{
Evaluation of co-trimoxazole in the treatment of multidrug-resistant tuberculosis
}

\author{
Noor Alsaad', Richard van Altena², Arianna D. Pranger ${ }^{1}$, Dick van Soolingen ${ }^{3,4}$, \\ Wiel C.M. de Lange ${ }^{2}$, Tjip S. van der Werf ${ }^{5}$, Jos G.W. Kosterink ${ }^{1}$ and \\ Jan-Willem C. Alffenaar ${ }^{1}$
}

Affiliations: 'Dept of Clinical Pharmacy, University Medical Centre Groningen, University of Groningen, Groningen, ${ }^{2}$ Tuberculosis Centre Beatrixoord, University Medical Centre Groningen, University of Groningen, Haren, ${ }^{3}$ Depts of Clinical Infection Diseases and Pulmonary Diseases and Medical Microbiology, Radboud University Nijmegen Medical Centre, Nijmegen, ${ }^{4}$ National Tuberculosis Reference Laboratory, National Institute for Public Health and the Environment (RIVM). Bilthoven, and ${ }^{5}$ Depts of Internal Medicine and Pulmonary Diseases and Tuberculosis, University Medical Centre Groningen, University of Groningen, Groningen, The Netherlands.

Correspondence: J.W.C Alffenaar, Dept of Hospital and Clinical Pharmacy, University Medical Center Groningen, University of Groningen, PO Box 30.001, 9700 RB Groningen, The Netherlands. E-mail: j.w.c.alffenaardumcg.nl

ABSTRACT Co-trimoxazole (SXT), a combination of sulfamethoxazole and trimethoprim, has shown in vitro activity against Mycobacterium tuberculosis. However, the pharmacokinetic and pharmacodynamic parameters of SXT in multidrug-resistant (MDR) tuberculosis (TB) are, thus far, lacking. Therefore, we evaluated its pharmacokinetics and drug susceptibility, along with its tolerability during treatment.

Based on drug susceptibility testing, MDR-TB patients received SXT as a part of their MDR treatment. The pharmacokinetic parameters of sulfamethoxazole, the effective component of SXT against M. tuberculosis, were evaluated. The ratio of the area under the curve from 0 to $24 \mathrm{~h}$ (AUC0-24) to minimum inhibitory concentration (MIC) was used as the best pharmacokinetic/pharmacodynamic parameter to predict the efficacy of sulfamethoxazole. Adverse effects of SXT were also evaluated.

10 patients with MDR-TB (one of whom had extensively drug-resistant TB) received $480 \mathrm{mg}$ of SXT with a median dosage of $6.5 \mathrm{mg} \cdot \mathrm{kg}^{-1}$ of SXT (range $6.1-6.8 \mathrm{mg} \cdot \mathrm{kg}^{-1}$ ) once daily for a median treatment period of 381 days (range 129-465 days). In two patients, the dose was escalated to $960 \mathrm{mg}$. The free AUC0-24/MIC of sulfamethoxazole exceeded 25 in only one patient. SXT was safe and well-tolerated, except for one patient who had gastrointestinal side-effects after receiving $960 \mathrm{mg}$ of SXT. Additional studies are needed to find the pharmacokinetic and pharmacodynamic targets, and consequently to set the optimal dose, of SXT for MDR-TB treatment.

@ERSpublications

Co-trimoxazole was found to be safe and well tolerated in 10 patients with multidrug-resistant tuberculosis http://ow.ly/lLrR0

Received: July 232012 | Accepted after revision: Sept 192012 | First published online: Oct 252012

Conflict of interest: Disclosures can be found alongside the online version of this article at www.erj.ersjournals.com 


\section{Introduction}

Multidrug-resistant (MDR) and extensively drug-resistant (XDR) tuberculosis (TB) are emerging in many areas around the world [1]. In addition, the susceptibility of Mycobacterium tuberculosis to current anti-TB drugs has decreased and, therefore, the treatment of MDR-TB has become increasingly complicated [2]. Consequently, there is an urgent need for new effective drugs with minimal toxicity. An old, inexpensive and well-tolerated drug like co-trimoxazole (SXT), which is registered for other indications than TB, could be a new effective agent for the treatment of MDR- and XDR-TB [3].

SXT is a combination of trimethoprim (TMP) and sulfamethoxazole (SMX) in a ratio of 1:5. It is a potent antibacterial drug against a variety of pathogens causing infections in humans. SXT is currently used in the treatment of urinary tract infections, otitis media, shigellosis, chronic bronchitis and Pneumocystis carinii pneumonitis [4].

SXT shows concentration-independent or time-dependent killing. Therefore, the ratio of area under the free concentration-time curve from 0 to $24 \mathrm{~h}$ ( $f$ AUC0-24) relative to the minimum inhibitory concentration (MIC) is considered to be the important pharmacokinetic/pharmcodynamic parameter in predicting the efficacy of SXT [5]. Very little is known about the pharmacodynamics of SXT [5]. There are only a few publications on the pharmacokinetic parameters of SXT, none of which include TB patients (table 1) [6-9]. Only two studies investigated the in vitro susceptibility of SXT against $M$. tuberculosis and showed promising results $[3,10]$. From these studies it could be concluded that only SMX is effective against $M$. tuberculosis, and that TMP is not $[3,10,11]$. In one study an SXT AUC0-24/MIC ratio of $>25$ was necessary for the effective treatment of melioidosis caused by Burkholderia pseudomallei [12]. The lack of data is probably due to the fact that SXT is an old drug and in vitro evaluation of pharmacokinetic and pharmacodynamic parameters in infection models is rather new.

In general, SXT is a safe and well-tolerated drug. Gastrointestinal complications, including nausea, vomiting, anorexia and diarrhoea are the most common adverse effects of SMX [13, 14]. Renal side-effects, including hyperkalaemia, a slight increase in the serum creatinine level and hyponatraemia, occur, especially in patients with renal dysfunction [14-16]. Other side-effects reported are haematological side-effects such as megaloblastic anaemia, leukopenia, thrombocytopenia and aplastic anaemia in patients with pre-existing megaloblastic anaemia or deficiencies in folic acid stores (alcoholics, malnourished patients and pregnant females) [17].

Although SXT has been administered to TB patients, data is very scarce and its role in TB treatment is still not yet clear. The objective of this study was to evaluate the pharmacokinetics, pharmacodynamics and pharmacokinetic/pharmacodynamic parameters and safety/tolerability of SXT in MDR-TB patients

\section{Methods \\ Patients}

MDR-TB patients who were referred to the Tuberculosis Centre Beatrixoord of the University Medical Centre Groningen (Groningen, the Netherlands) between January 1, 2006 and July 1, 2012, and for whom drug susceptibility testing for SXT was performed, were eligible for evaluation. Age, sex, weight, ethnicity, underlying disease, MIC of the M. tuberculosis isolate, localisation of TB, other anti-TB medications, duration of treatment with SXT and the total anti-TB regimen administration were recorded for MDR-TB patients who received SXT. Patients were subjected to routine medical care without specific study-related interventions. We describe patient data obtained during usual care and, therefore, no ethical clearance was required under Dutch law.

\section{TABLE 1 Pharmacokinetic parameters of sulfamethoxazole for the treatment of different infections from previous studies}

\begin{tabular}{|c|c|c|c|c|c|c|c|}
\hline Infection & Subjects & Dosage regimen & $t_{1 / 2} h$ & $V D L \cdot k^{-1}$ & $\begin{array}{c}\text { Clearance } \\
\mathrm{mL} \cdot \mathrm{min}^{-1} \cdot \mathbf{k g}^{-1}\end{array}$ & $\begin{array}{c}\text { AUC } \\
\mathrm{mg} \cdot \mathrm{L}^{-1} \cdot \mathrm{h}^{-1}\end{array}$ & [Ref.] \\
\hline Bacterial skin disease & 12 & Single dose $0.23 \mathrm{~g}$ orally & $10.0 \pm 1.1$ & $\mathrm{~N} / \mathrm{A}$ & $\mathrm{N} / \mathrm{A}$ & $1295 \pm 823$ & [6] \\
\hline AIDS & 8 & $75 \mathrm{mg} \cdot \mathrm{kg}^{-1}$ daily i.v. & $15.5 \pm 7.4$ & $0.5 \pm 0.3$ & $0.40 \pm 0.12$ & $\mathrm{~N} / \mathrm{A}$ & [8] \\
\hline HIV & 10 & $800 \mathrm{mg}$ once daily orally & $\mathrm{N} / \mathrm{A}$ & $\mathrm{N} / \mathrm{A}$ & $\mathrm{N} / \mathrm{A}$ & $574.2(342.6-796.3)$ & [9] \\
\hline
\end{tabular}

Data are presented as $n$, mean \pm SD or median (range). $t_{1 / 2}$ : half-life; VD: volume of distribution; AUC: area under the concentration-time curve; N/A: not available. 


\section{Pharmacokinetics and pharmacodynamics}

Blood samples were only evaluated when obtained at steady state, which was after $\geqslant 3$ days of administration of SXT [18]. They were collected before and at 1, 2, 3, 4 and $8 \mathrm{~h}$ after SXT administration.

The concentrations of SMX in human plasma samples were analysed in the Clinical Toxicology and Drugs Analysis laboratory of the Department of Hospital and Clinical Pharmacy at the University Medical Centre Groningen by a validated liquid chromatography-tandem mass spectrometry. In brief, $5 \mu \mathrm{L}$ of each plasma sample was mixed with $750 \mu \mathrm{L}$ precipitation reagent (methanol and acetonitrile 4:21 volume/volume). From the clear upper layer $5 \mu \mathrm{L}$ was injected on a $50 \times 2.1 \mathrm{~mm}$ reversed phase $\mathrm{C} 18,5-\mu \mathrm{m}$ analytical column (HyPurity Aquastar; Interscience, Breda, the Netherlands) for chromatographic separation. The detector was operated in electrospray positive ionisation mode and performed selected reaction monitoring as the scanning mode. The transition of $\mathrm{m} / z$ 254.1-155.9 (collision energy $25 \mathrm{eV}$ ) for SMX was measured with scan width of $0.5 \mathrm{~m} / z$. The recoveries ranged from $97.7 \%$ to $102.9 \%$, depending on the concentration. Accuracy was between $97.7 \%$ and $102.9 \%$ for SMX, depending on the concentration level. The intra- and inter-assay coefficients of variation were $<5.1 \%$ over the ranges of $5-100 \mathrm{mg} \cdot \mathrm{L}^{-1}$. The lower limit of quantisation was $5 \mathrm{mg} \cdot \mathrm{L}^{-1}$.

Pharmacokinetic parameters of SMX, including AUC0-24, volume of distribution ( VD), clearance $(\mathrm{Cl})$ and half-life $\left(t_{1 / 2}\right)$ were determined with a standard noncompartmental pharmacokinetic method using the KINFIT module of MW/Pharm 3.60 (Mediware, Groningen, the Netherlands). The AUC0-24 in plasma was calculated according to the log-linear trapezoidal rule. In this case, the concentration of SMX at $24 \mathrm{~h}$ after oral administration was estimated to be equal to its concentrations at time $0 \mathrm{~h}$ (before administration of the dose).

The free nonprotein-bound fraction of antibacterial drug is responsible for the clinical effect of these drugs [19]. The free AUC0-24 ( $f$ AUC0-24) values were estimated by multiplying the total AUC0-24 values by the unbound fraction of 0.23 , which was retrieved from an earlier study [19]. Free-drug AUC0-24/MIC ratios were calculated by dividing the $\mathrm{AAUC} 0-24$ by the MIC value for SMX.

A one-compartmental pharmacokinetic population model (POP-PK) of SMX with first-order absorption without lag time was developed using an iterative two-stage Bayesian procedure (MW/Pharm 3.60) starting with pharmacokinetic estimates from a previous study [9]. The individual pharmacokinetic parameters of each patient were calculated using KINFIT. KINPOP is used to calculate the parameters of the MDR-TB patients based on the individual concentrations of SMX and patient characteristics such as body weight, age, sex and creatinine clearance [20].

To determine MIC values, M. tuberculosis isolates were subjected to drug susceptibility testing, which was performed on the Middlebrook 7H10 agar (Becton Dickinson and Company, Sparks, MD, USA) dilution method at the Dutch National Mycobacterium Reference Laboratory (National Institute for Public Health and the Environment (RIVM), Bilthoven, the Netherlands) [21]. In accordance with European Committee on Antimicrobial Susceptibility Testing guidelines, the MIC of SXT is expressed as TMP:SMX in the ratio 1:19.

\section{Safety}

The safety of SXT during TB treatment was evaluated by assessing the reported side-effects of SXT retrospectively using a standardised data abstraction form from the Tuberculosis Centre Beatrixoord. Specific attention was paid to the side-effects that could be caused by SXT, such as gastrointestinal sideeffects (nausea, vomiting and diarrhoea), hepatotoxicity and anaemia and blood count abnormalities [22].

Hepatic injury was defined as an elevation in one of the hepatic enzymes five times the upper limit of normal during treatment with SXT (grade 3 common toxicity criteria ). These enzymes include aspartate aminotransferase (ASAT) $\left(>200 \mathrm{U} \cdot \mathrm{L}^{-1}\right)$, alanine aminotransferase (ALAT) $\left(>225 \mathrm{U} \cdot \mathrm{L}^{-1}\right)$ and $\gamma$-glutamyl transpeptidase $\left(>200-275 \mathrm{U} \cdot \mathrm{L}^{-1}\right)$ [23]. For other side-effects the defined normal values were: anaemia (haemoglobin normal range $7.5-9.9 \mathrm{mmol} \cdot \mathrm{L}^{-1}$ (female) and $8.7-10.6 \mathrm{mmol} \cdot \mathrm{L}^{-1}$ (male)), leukocyte count $\left(4 \times 10^{9} \cdot \mathrm{L}^{-1}\right)$ and platelet count $\left(150-350 \times 10^{9} \cdot \mathrm{L}^{-1}\right)$. A standard causality assessment tool (Naranjo algorithm) is required for adverse drug reactions (ADRs) in patients on multiple drug regimens, like MDRTB patients [24]. Using this tool, the likelihood that SXT caused ADRs was scored as: $\geqslant 9$ definite, 5-8 probable, $1-4$ possible, $\leqslant 0$ doubtful.

\section{Statistical analysis}

Wilcoxon signed-rank test was employed in the statistical analysis when the data were not normally distributed. The POP-PK model was cross-validated by developing a POP-PK model based on n-1 and by predicting the AUC0-24 of the subject left out during the model development. The correlation between the predicted AUC0-24 value based on the POP-PK model and calculated AUC0-24 was tested by means of Bland-Altman analysis. 


\begin{abstract}
Results
Patients

Drug susceptibility testing for SXT was performed on 17 MDR-TB patients. SXT was used as part of the TB regimen in only 10 patients because drug susceptibility testing showed that isolates were susceptible to SXT. In the other cases, resistance to SXT $(n=4)$ or more conventional TB drugs could be used $(n=3)$ (table 2). In general, these TB patients were relativley young, with a median age of 29 years (interquartile range (IQR) 24-31 years), and had a relatively low median body mass index of $21.1 \mathrm{~kg} \cdot \mathrm{m}^{-2}\left(\right.$ IQR $\left.19.1-23.6 \mathrm{~kg} \cdot \mathrm{m}^{-2}\right)$. The resistance of $M$. tuberculosis to at least isoniazid and rifampicin was diagnosed by culture. Eight patients had pulmonary TB, the most common diagnosis in MDR-TB patients; one patient had urogenital TB; and one had both pulmonary and extrapulmonary TB. $480 \mathrm{mg}$ SXT daily was prescribed. This equals a median dose of $6.5 \mathrm{mg} \cdot \mathrm{kg}^{-1}$ (IQR $6.1-6.8 \mathrm{mg} \cdot \mathrm{kg}^{-1}$ ) in various combination regimens for a median period of 381 days (IQR 129-465 days). The daily dose of SXT was increased arbitrarily to $960 \mathrm{mg}\left(14\right.$ and $\left.13 \mathrm{mg} \cdot \mathrm{kg}^{-1}\right)$ in two patients, because of low levels of SXT in blood compared to MIC in these patients. All patients had a negative history for underlying diseases, except for one patient who had diabetes mellitus. None were diagnosed with co-infection with HIV. Eight of the 10 patients successfully completed the treatment with no signs of recurrence. Two patients are still undergoing treatment at the time of writing; sputum culture was converted and they are both in a good clinical condition. The clinical data of all 10 patients are shown in table 3. Drug susceptibility testing was evaluated in all MDR-TB patients. Susceptibility and resistance of M. tuberculosis to anti-TB drugs is shown in table 2.
\end{abstract}

\title{
Pharmacokinetics and pharmacodynamics
}

The steady-state pharmacokinetic parameters of SMX could be evaluated in only eight out of 10 patients receiving $480 \mathrm{mg}$ SXT once daily, because in the other two patients no plasma sampling was performed during the treatment period with SXT. The pharmacokinetic parameters are summarised in table 4 . The observed plasma concentration-time curves of SMX were obtained from the patients after receiving $480 \mathrm{mg}$ of SXT and are shown in figure 1.

TABLE 2 Susceptibility and resistance to anti-tuberculosis (TB) drugs in 17 multidrug-resistant patients

Resistance

Susceptibility

Intermediate susceptibility
$17(100)$

$14(82.4)$

$17(100)$

9 (53)

$13(76.5)$

7 (41.2)

$2(11.8)$

$15(88.2)$

$5(29.4)$

$1(5.9)$

$3(17.6)$

4 (23.5)

4 (23.5)

1 (5.9)

$13(76.5)$

2 (11.8)

9 (53)

2 (11.8)

4 (23.5)

7 (41.2)

7 (41.2)

1 (5.9)
2 (11.8)

8 (47)

$3(17.6)$

10 (58.8)

4 (23.5)

2 (11.8)

10 (58.8)

$13(76.5)$

$12(70.6)$

$12(70.6)$

7 (41.2)

3 (17.6)

11 (64.7)

5 (29.4)

4 (23.5)

13 (76.5)

1 (5.9)
1 (5.9)

1 (5.9)

Data are presented as $\mathrm{n}(\%)$. 


\begin{tabular}{|c|c|c|c|c|c|c|c|c|}
\hline 1 & M & 18.6 & 531 & 546 & Asian & $\begin{array}{l}\text { Smoking, } \\
\text { soft drugs }\end{array}$ & Pulmonary & $\begin{array}{c}\text { E, AM, MOX, PTH, } \\
\text { LZD, CFZ, Doxy }\end{array}$ \\
\hline 2 & $\mathrm{~F}$ & 21.5 & $191^{\circ}$ & $202^{\bullet}$ & African & None & $\begin{array}{l}\text { Pulmonary and } \\
\text { extrapulmonary }\end{array}$ & MOX, KM, ERTA \\
\hline 4 & $\mathrm{~F}$ & 18.1 & 412 & 548 & Asian & None & Pulmonary & $\begin{array}{c}\text { E, AM, MOX, PTH, } \\
\text { LZD, CFZ, CPX }\end{array}$ \\
\hline 5 & $\mathrm{~F}$ & 19.3 & 154 & 191 & Russian & None & Pulmonary & MOX, LZD, AMX/CL, ERTA \\
\hline 6 & M & 20.7 & 350 & 365 & Asian & Smoking & Pulmonary & $\begin{array}{c}\text { E, AM, MOX, LZD, } \\
\text { CFZ, CPX, Doxy }\end{array}$ \\
\hline 10 & M & 21.1 & $41^{\circ}$ & $44^{\circ}$ & African & None & Extrapulmonary & CLM, ERTA, LZD, KM \\
\hline
\end{tabular}

Data are presented as $n$, unless otherwise stated. BMI: body mass index; M: male; F: female; E: ethambutol; AM: amikacin; MOX: moxifloxacin; PTH: protionamide; LZD: linezolid; CFZ: clofazimine; Doxy: doxycycline; KM: kanamycin; ERTA: ertapenem; Z: pyrazinamide; CLM; clarithromycin; CM: capreomycin; CPX: ciprofloxacin; AMX/CL: amoxicillin + clavulanic acid (Augmentin); CS: cycloserine. ${ }^{\#}$ : taken at any time during MDR-TB treatment; ${ }^{\top}$ : patient still receiving treatment.

The parameters of the POP-PK model of SMX are shown in table 5. Cross-validation of this model showed that the geometric mean values of the POP-PK model (n-1) were $\mathrm{Cl} 1.28 \pm 0.52 \mathrm{~L} \cdot \mathrm{h}^{-1} / 1.85 \mathrm{~m}^{2}$; VD $0.22 \pm 0.03 \mathrm{~L} \cdot \mathrm{kg}^{-1}$ lean body mass; and absorption rate constant $\left(0.44 \pm 0.18 \mathrm{~h}^{-1}\right)$. These results were not different from the POP-PK model (table 5). The individual difference between the predicted values based on the POP-PK model and calculated values of AUC0-24 was underestimated by a median percentage of -0.7 (range -6.2-2.8). The agreement between predicted using the POP-PK model and calculated AUC0-24 value of SMX is shown in figure 2. This figure shows that the values of AUC0-24 were within agreement; only one was outside this agreement. The median percentage of difference between the predicted based on the POPPK (n-1) model and calculated values of AUC0-24 was -3.92 (range -6.3-1.7).

Drug susceptibility testing shows that MIC values of SMX for M. tuberculosis varied with median ranges of $9.5 \mathrm{mg} \cdot \mathrm{L}^{-1}\left(\mathrm{IQR} 4.8-25 \mathrm{mg} \cdot \mathrm{L}^{-1}\right)$.

The ratios of $f$ AUC0-24/MIC of SMX in each patient are presented in table 6. The geometric means of AUC0-24/MIC and fAUC0-24/MIC ratios after receiving $480 \mathrm{mg}$ of SMX were 48.4 (IQR 34.8-71.3) and 11.1 (IQR 8-16.4), respectively. One of the eight patients who received $480 \mathrm{mg}$ of SXT had a $f$ AUC0-24/MIC ratio of $\mathrm{SMX}>25$.

\section{Safety}

In general, SXT was well tolerated. However, there were some mild side-effects, including abdominal complaints with diarrhoea and vomiting in one patient (Naranjo score $=4$ ). Elevations in hepatic enzymes (ASAT and ALAT) were observed in two patients who were receiving 480 and $960 \mathrm{mg}$ of SXT, respectively

\begin{tabular}{|c|c|}
\hline$A U C_{0-24} \mathrm{mg} \cdot h \cdot \mathrm{L}^{-1}$ & $371.5(360-574.8)$ \\
\hline Clearance $\mathrm{mL} \cdot \mathrm{min}^{-1} \cdot \mathrm{kg}^{-1}$ & $0.19(0.14-0.25)$ \\
\hline VD L $\cdot \mathbf{k g}^{-1}$ & $0.15(0.13-0.22)$ \\
\hline$t_{1 / 2} h$ & $10.1(8.7-10.8)$ \\
\hline
\end{tabular}

Data are presented as median (interquartile range). AUC0-24: area under the concentration-time curve up to $24 \mathrm{~h}$ post-dosage; VD: volume of distribution; $t_{1 / 2}$ : half-life. ${ }^{\#}: \mathrm{n}=8$. 


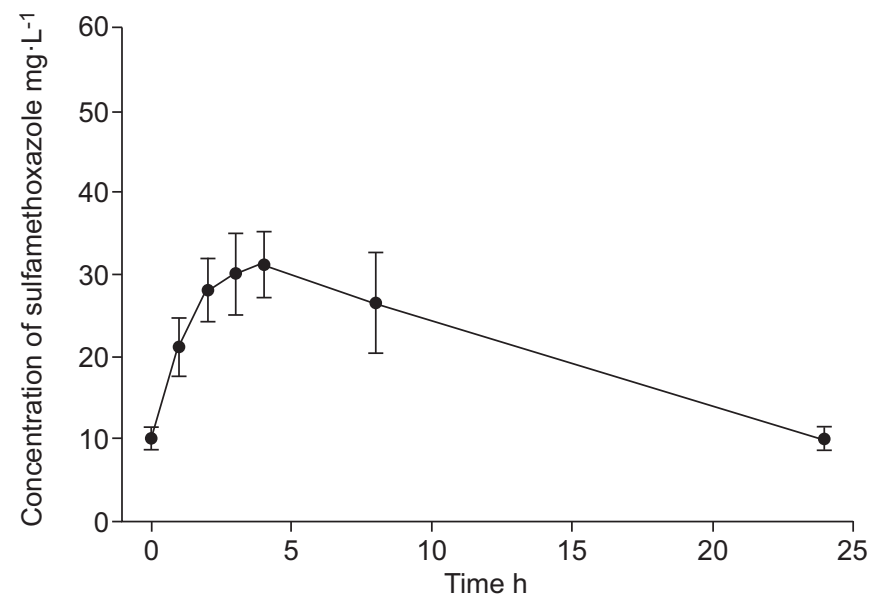

FIGURE 1 Plasma concentration-time curves of sulfamethoxazole. $n=8$.

(Naranjo score $=5$ in both cases). In these two patients the values of ALAT during treatment with $480 \mathrm{mg}$ SXT were 47.50 and $48 \mathrm{U} \cdot \mathrm{L}^{-1}$ (baseline $29 \mathrm{U} \cdot \mathrm{L}^{-1}$ ) and with $960 \mathrm{mg}$ of SXT were 46 and $71 \mathrm{U} \cdot \mathrm{L}^{-1}$ (baseline $\left.43 \mathrm{U} \cdot \mathrm{L}^{-1}\right)$, respectively. The values of ASAT after receiving 480 and $960 \mathrm{mg}$ of SXT were 48 and $99 \mathrm{U} \cdot \mathrm{L}^{-1}$, respectively, compared with a baseline of $35 \mathrm{U} \cdot \mathrm{L}^{-1}$. Although the hepatic enzymes were two times higher than baseline, the values of ASAT and ALAT did not exceed five times the upper limit of normal. The median haemoglobin level in patients before treatment with $480 \mathrm{mg}$ SXT was $7.6 \mathrm{mmol} \cdot \mathrm{L}^{-1}$ (range 7.6 $8 \mathrm{mmol} \cdot \mathrm{L}^{-1}$ ) and during treatment was $7 \mathrm{mmol} \cdot \mathrm{L}^{-1}\left(\right.$ range $6.6-7.2 \mathrm{mmol} \cdot \mathrm{L}^{-1}$ ); the difference was significant $(\mathrm{p}=0.002)$ but probably clinically not relevant. The Naranjo score value for low haemoglobin level is 3 or 4 . One patient developed leukocytopaenia after receiving 480 and $960 \mathrm{mg}$ of SXT. Leukocyte counts were $2.2 \times 10^{9}$ cells $\cdot \mathrm{L}^{-1}$ and $3.2 \times 10^{9}$ cells $\cdot \mathrm{L}^{-1}$, respectively, in comparison to a baseline value of 4.2 cells $\cdot \mathrm{L}^{-1}$ (Naranjo score $=3$ ). One patient developed mild thrombocytopaenia with a thrombocyte count of $146 \times 10^{9}$ cells $\cdot \mathrm{L}^{-1}\left(247 \times 10^{9}\right.$ cells $\cdot \mathrm{L}^{-1}$ at baseline $)($ Naranjo score $=3)$.

\section{Discussion}

No previous study has described the pharmacokinetic, pharmacodynamic and pharmacokinetic/ pharmacodynamic parameters of SXT in MDR-TB patients. It is interesting that the pharmacokinetic parameters of SMX in TB patients, including AUC0-24, VD and clearance, are lower than the values observed in patients with meningitis, HIV infection or those suffering from bacterial skin infection (table 1) [6-9]. Low drug exposure may be explained by decreased intestinal absorption resulting in low serum concentrations of anti-TB drugs. Other factors such as alcohol abuse, smoking, weight loss, low albumin and haemoglobin levelscould be the possible reasons for reduced permeability via paracellular intestinal transport [25]. To further explore the pharmacokinetic parameters in MDR-TB patients, we made a population model. This model showed no significant differences $(p=0.78)$ between the calculated and predicted AUC0-24 according to Bland-Altman analysis. Thus, MDR-TB patients seem to display a consistent pharmacokinetic profile for SMX. Therefore, the model developed could be used to assess drug exposure in a prospective study to evaluate the safety and efficacy and to find the most suitable dose of SXT as part of a TB treatment regimen.

The MIC value of SMX in this study was in accordance with a previous study that stated that SMX inhibits $80 \%$ of growth of all 117 isolates at MIC of $19 \mathrm{mg} \cdot \mathrm{L}^{-1}[10]$.

The pharmacokinetic/pharmacodynamic parameter (AUC0-24/MIC) best predicting SMX efficacy has not been firmly established. According to a single earlier study, the $f$ AUC0-24/MIC of SMX had to be $>25$ for

TABLE 5 Population pharmacokinetic model parameter values of sulfamethoxazole

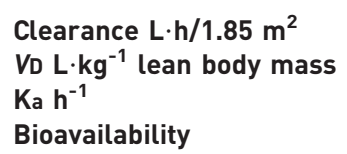

$1.14 \pm 0.43$

$0.24 \pm 0.05$

$0.43 \pm 0.17$

1

Data are presented as geometric mean \pm SD. VD: volume of distribution; Ka: absorption rate constant. 


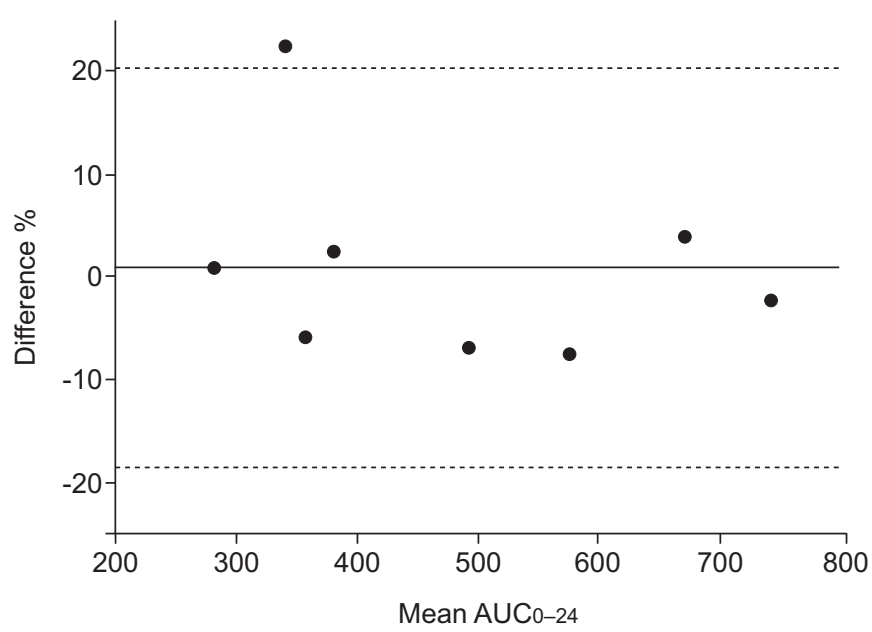

FIGURE 2 Bland-Altman plot showing the agreement between predicted and calculated area under the concentrationtime curve up to $24 \mathrm{~h}$ post-dosage (AUC0-24) of sulfamethoxazole.

adequate treatment of melioidiosis [12]. From the results, it can be seen that in only one out of eight patients was the fAUC0-24/MIC ratio of SMX $>25$. However, this ratio could be $<25$ and still be effective in TB treatment. In particular, the magnitude of this parameter may vary for different bacterial species. For example the AUC0-24/MIC ratio for fluoroquinolone is different against Pseudomonas aeruginosa and other Gram-negative bacilli [26]. The lower ratio might be acceptable in patients receiving multidrug treatment for MDR-TB. Other drugs might decrease this ratio, as shown in a murine aerosol infection model from a previous study, in which the AUC0-24/MIC ratio of rifampicin that correlated with efficacy decreased when administered in combination with moxifloxacin [27]. Therefore the interpretation of the value of the AUC0-24/MIC for SMX in our MDR-TB patients at this time is difficult. The dose of SXT given to the MDR-TB patients was low compared to commonly used dosages for other infectious diseases. However, the more conventional dosages of SXT are for the treatment of fast-replicating bacteria and are often given as monotherapy. In our case, the target is slow-growing $M$. tuberculosis, in combination with other antimicrobial agents. For future studies of SXT for MDR-TB we advise exploring higher dosages in order to achieve higher drug exposure, but tolerability may be a problem during prolonged treatment.

TB patients with HIV co-infection can have drug-drug interaction when rifampicin is co-administered with SXT, as mentioned in a previous study [9], but this is of no concern in MDR-TB. Indeed, rifampicin decreases concentrations of TMP and SMX significantly in serum, but SXT would not be prescribed in individuals who can be treated with rifampicin, and none had received rifampicin before starting the treatment with SXT.

TABLE 6 Ratio of protein unbound (free $(f)$ ) area under the concentration-time curve from 0 to $24 \mathrm{~h}$ post-dosage $\left(\mathrm{AUC}_{0}-24\right)$ relative to the minimum inhibitory concentration (MIC) of sulfamethoxazole (SMX) after receiving $480 \mathrm{mg}$ and $960 \mathrm{mg}$ of co-trimoxazole ${ }^{\#}$

Patient

MIC $\mathrm{mg} \cdot \mathrm{L}^{-1}$

SMX $400 \mathrm{mg}$

SMX $800 \mathrm{mg}$

\begin{tabular}{cccc}
\hline $\begin{array}{c}A U C 0-24 \\
\mathrm{mg} \cdot \mathrm{h} \cdot \mathrm{L}^{-1}\end{array}$ & fAUC0-24/MIC & $\begin{array}{c}\text { AUC } 0-24 \\
\mathrm{mg} \cdot \mathrm{h} \cdot \mathrm{L}^{-1}\end{array}$ & fAUC $0-24 / \mathrm{MIC}$
\end{tabular}

\begin{tabular}{lccccc}
\hline $\mathbf{1}$ & 25 & 376 & 3 & 774 & 7 \\
$\mathbf{2}$ & 4.75 & 297 & 14 & & \\
$\mathbf{5}$ & 9.5 & 658 & 16 & 991 & \\
$\mathbf{6}$ & 25 & 509 & 5 & \\
$\mathbf{7}$ & 4.75 & 597 & 29 & \\
$\mathbf{8}$ & 4.75 & 367 & 18 & \\
$\mathbf{9}$ & 19 & 752 & 9 & \\
$\mathbf{1 0}$ & 4.75 & 281 & 14 & & \\
\hline
\end{tabular}

Data are presented as $n .{ }^{*}: \mathrm{n}=8$. 
Although our sample size was low, this retrospective study confirmed the safety of SXT in accordance with earlier studies that showed that SXT was safe and well tolerated when it was used as prophylaxis in adults with HIV infection who have pulmonary TB $[28,29]$. SXT was well tolerated in MDR-TB patients and was not discontinued in any of the eight patients before the end of treatment and in the two patients who are still undergoing treatment. Only one patient had gastrointestinal complaints as a possible side-effect of SXT following administration of $960 \mathrm{mg}$ daily. Therefore, the dose was lowered to $480 \mathrm{mg}$ until the end of treatment. In our patients, the maximum Naranjo score of 5 was reached, in other words there is a probable relationship between the observed side-effects and SXT. However, haematological side-effects, including anaemia, leukocytopaenia and thrombocytopaenia, during treatment could be due to other anti-TB drugs such as linezolid [30, 31].

The main limitation of this study is that there is a lack of data on the target (AUC0-24/MIC value) to be reached to predict the efficacy of SXT in the treatment of TB. In future, a prospective study is needed to evaluate the pharmacokinetic and pharmacodynamic parameters of SXT in TB patients. To determine the fAUC0-24/ MIC ratio of SMX for effective treatment along with suppression of the emergence of drug resistance, an in vitro infection model could be the suitable strategy, as reported previously for moxifloxacin [32].

SXT, in comparison with other drugs not registered for TB treatment but used in MDR-TB regimens, has the great advantage that it is cheap and readily available all over the world.

These are the first results on the inclusion of SXT in MDR-TB treatment in which drug susceptibility testing and SXT concentration measurements were combined. Based on our preliminary data we showed that SXT has a favourable pharmacokinetic profile in TB patients.

Further in vitro pharmacokinetic and pharmacodynamic studies, such as a hollow fibre infection model or mouse model, are warranted to establish target AUC0-24/MIC values to predict the efficacy of SXT, and consequently to set the optimal dose in the treatment of MDR-TB treatment. Preferably, pharmacokinetic/ pharmacodynamic parameters of SXT and MIC of SXT should be measured alone and in the presence of other anti-TB drugs to detect the possible synergism between these drugs. According to the clinical outcome, which showed no treatment discontinuation or serious side-effects, the consistent pharmacokinetic values and relative low MIC values, this study could be the starting point for further exploration of SXT for MDR-TB treatment.

\section{Acknowledgements}

We would like to thank the nursing staff at the Tuberculosis Centre Beatrixoord (Haren, the Netherlands) for their cooperation in data collection from patients, and also the patients who volunteered to help obtain these data.

\section{References}

1 Caminero JA. Treatment of multidrug-resistant tuberculosis: evidence and controversies. Int J Tuberc Lung Dis 2006; 10: 829-837.

2 Alffenaar JW, van Altena R, Harmelink IM, et al. Comparison of the pharmacokinetics of two dosage regimens of linezolid in multidrug-resistant and extensively drug-resistant tuberculosis patients. Clin Pharmacokinet 2010; 49: 559-565.

3 Forgacs P, Wengenack NL, Hall L, et al. Tuberculosis and trimethoprim-sulfamethoxazole. Antimicrob Agents Chemother 2009; 53: 4789-4793.

4 De Angelis DV, Woolley JL, Sigel CW. High-performance liquid chromatographic assay for the simultaneous measurement of trimethoprim and sulfamethoxazole in plasma or urine. Ther Drug Monit 1990; 12: 382-392.

5 Nightingale $\mathrm{CH}$, Ambrose P, File TM Jr, eds. Community Acquired Respiratory Infections. New York, Marcel Dekker, Inc., 2003.

6 Krolicki A, Klimowicz A, Bielecka-Grzela S, et al. Penetration of cotrimoxazole components into skin after a single oral dose. Theoretical versus experimental approach. Pol J Pharmacol 2004; 56: 257-263.

7 Dudley MN, Levitz RE, Quintiliani R, et al. Pharmacokinetics of trimethoprim and sulfamethoxazole in serum and cerebrospinal fluid of adult patients with normal meninges. Antimicrob Agents Chemother 1984; 26: 811-814.

8 Chin TW, Vandenbroucke A, Fong IW. Pharmacokinetics of trimethoprim-sulfamethoxazole in critically ill and non-critically ill AIDS patients. Antimicrob Agents Chemother 1995; 39: 28-33.

9 Ribera E, Pou L, Fernandez-Sola A, et al. Rifampin reduces concentrations of trimethoprim and sulfamethoxazole in serum in human immunodeficiency virus-infected patients. Antimicrob Agents Chemother 2001; 45: 3238-3241.

10 Huang TS, Kunin CM, Yan BS, et al. Susceptibility of Mycobacterium tuberculosis to sulfamethoxazole, trimethoprim and their combination over a 12 year period in Taiwan. J Antimicrob Chemother 2012; 67: 633-637.

11 Ong W, Sievers A, Leslie DE. Mycobacterium tuberculosis and sulfamethoxazole susceptibility. Antimicrob Agents Chemother 2010; 54: 2748.

12 Cheng AC, McBryde ES, Wuthiekanun V, et al. Dosing regimens of cotrimoxazole (trimethoprimsulfamethoxazole) for melioidosis. Antimicrob Agents Chemother 2009; 53: 4193-4199.

Masters PA, O’Bryan TA, Zurlo J, et al. Trimethoprim-sulfamethoxazole revisited. Arch Intern Med 2003; 163: 402-410. Smilack JD. Trimethoprim-sulfamethoxazole. Mayo Clin Proc 1999; 74: 730-734.

Masters PA, O'Bryan TA, Zurlo J, et al. Trimethoprim-sulfamethoxazole revisited. Arch Intern Med 2003; 163: 402-410. Mori H, Kuroda Y, Imamura S, et al. Hyponatremia and/or hyperkalemia in patients treated with the standard dose of trimethoprim-sulfamethoxazole. Intern Med 2003; 42: 665-669. 

updated: June 14, 2010. Date last accessed: May 2012.

24 Naranjo CA, Busto U, Sellers EM, et al. A method for estimating the probability of adverse drug reactions. Clin Pharmacol Ther 1981; 30: 239-245.

25 Barroso EC, Pinheiro VG, Facanha MC, et al. Serum concentrations of rifampin, isoniazid, and intestinal absorption, permeability in patients with multidrug resistant tuberculosis. Am J Trop Med Hyg 2009; 81: 322-329.

26 Nuermberger E, Grosset J. Pharmacokinetic and pharmacodynamic issues in the treatment of mycobacterial infections. Eur J Clin Microbiol Infect Dis 2004; 23: 243-255.

27 Balasubramanian V, Solapure S, Gaonkar S, et al. Effect of coadministration of moxifloxacin and rifampin on Mycobacterium tuberculosis in a murine aerosol infection model. Antimicrob Agents Chemother 2012; 56: 3054-3057.

28 Wiktor SZ, Sassan-Morokro M, Grant AD, et al. Efficacy of trimethoprim-sulphamethoxazole prophylaxis to decrease morbidity and mortality in HIV-1-infected patients with tuberculosis in Abidjan, Cote d'Ivoire: a randomised controlled trial. Lancet 1999; 353: 1469-1475.

29 Nunn AJ, Mwaba P, Chintu C, et al. Role of co-trimoxazole prophylaxis in reducing mortality in HIV infected adults being treated for tuberculosis: randomised clinical trial. BMJ 2008; 337: a257.

30 Gerson SL, Kaplan SL, Bruss JB, et al. Hematologic effects of linezolid: summary of clinical experience. Antimicrob Agents Chemother 2002; 46: 2723-2726.

31 von der Lippe B, Sandven P, Brubakk O. Efficacy and safety of linezolid in multidrug resistant tuberculosis (MDRTB) - a report of ten cases. J Infect 2006; 52: 92-96.

32 Gumbo T, Louie A, Deziel MR, et al. Selection of a moxifloxacin dose that suppresses drug resistance in Mycobacterium tuberculosis, by use of an in vitro pharmacodynamic infection model and mathematical modeling. J Infect Dis 2004; 190: 1642-1651. 